\title{
Initial Clinical Status and Spot Sign Are Associated with Intraoperative Aneurysm Rupture in Patients Undergoing Surgical Clipping for Aneurysmal Subarachnoid Hemorrhage
}

\author{
Jan-Karl Burkhardt ${ }^{1, *}$ Marian Christoph Neidert ${ }^{1, *}$ Malte Mohme ${ }^{1} \quad$ Burkhardt Seifert $^{2} \quad$ Luca Regli $^{1}$ \\ Oliver Bozinov ${ }^{1}$ \\ ${ }^{1}$ Department of Neurosurgery, University Hospital Zurich, University \\ of Zurich, Zurich, Switzerland \\ Address for correspondence Jan-Karl Burkhardt, MD, Department of \\ Neurosurgery, University Hospital Zurich, Frauenklinikstrasse 10, \\ ${ }^{2}$ Division of Biostatistics, University of Zurich, Institute for Social and \\ Zurich CH-8091, Switzerland (e-mail: Jan-Karl.Burkhardt@usz.ch). \\ Preventive Medicine, Zurich, Switzerland \\ *Dr. Burkhardt and Dr. Neidert contributed equally to the article.
}

J Neurol Surg A 2016;77:130-138.

\begin{abstract}
Keywords

- intracerebral hemorrhage

- spot sign

- intraoperative aneurysm rupture

- surgical clipping

- aneurysmal subarachnoid hemorrhage

Objective To assess clinical and radiographic risk factors for intraoperative aneurysm rupture (ioAR) during surgical clipping after aneurysmal subarachnoid hemorrhage (aSAH) and to analyze its influence on patient outcome.

Methods Patient selection was based on a retrospective analysis of our prospective subarachnoid hemorrhage patient database including consecutive patients between January 2008 and August 2012 with aSAH undergoing microsurgical clipping. Demographic data, cardiovascular risk factors, preoperative radiologic aneurysm characteristics, as well as timing of surgery and preoperative severity grades (Hunt and Hess [HH], Fisher, World Federation of Neurological Societies [WFNS]), were collected from hospital charts and surgery videos and compared between patients with and without ioAR.

Results Of 100 patients ( 38 men, 62 women) with a median age of 57.4 years (range: 23-85 years), ioAR occurred in 34 cases (34\%). Univariate analyses showed that severity grades were significantly higher in the ioAR group (Fisher $p=0.012 ; \mathrm{HH} p=0.002$; WFNS $p=0.023$ ). IOAR was significantly associated with intracerebral hemorrhage (ICH) $(23 \%$ versus $47 \% ; p=0.013)$ and the spot sign as an indicator of active bleeding within the $\mathrm{ICH}(0 \%$ vs $44 \% ; p=0.007)$. Multivariate analysis showed that $\mathrm{HH}$ was the only significant predictor of ioAR ( $p=0.03$; odds ratio: 2.3 ; $95 \%$ confidence interval, 1.1-5.0). With a mean follow-up of 17.6 months $( \pm 16.6)$, Glasgow Outcome Scale score, mortality rate ( $12 \%$ versus $15 \% ; p=0.82$ ), delayed cerebral ischemia ( $36 \%$ versus $38 \% ; p=0.51$ ), and shunt dependency ( $32 \%$ versus $44 \% ; p=0.23$ ) were comparable between the non-ioAR and ioAR group.

Conclusions Initial clinical status and spot sign were associated with ioAR during microsurgical clipping of ruptured aneurysms. However, there was no difference regarding clinical outcome and complications of the two groups.
\end{abstract}

received

June 29, 2014

accepted after revision

April 24, 2015

published online

July 27, 2015 (c) 2016 Georg Thieme Verlag KG

Stuttgart · New York
DOI http://dx.doi.org/ 10.1055/s-0035-1558414. ISSN 2193-6315. 


\section{Introduction}

Aneurysmal subarachnoid hemorrhage (aSAH) accounts for $\sim 5 \%$ of all strokes and affects $\sim 30,000$ people in the United States per year. ${ }^{1,2}$ With an overall mortality of $\sim 45 \%$, aSAH is a major contributor to the stroke-related loss of productive life years because it occurs at younger ages than ischemic stroke or intracerebral hemorrhage (ICH). ${ }^{3-6}$ In addition to the primary bleeding event and delayed ischemic neurologic deficits, early rebleeding from ruptured intracranial aneurysms is a devastating complication associated with high mortality and morbidity. ${ }^{7}$ The prevention of rebleeding and its associated complications is the main rationale of early surgical or interventional aneurysm treatment. During surgical clipping of ruptured aneurysms, rebleeding is referred to as intraoperative aneurysm rupture (ioAR). Rates of ioAR in the literature range from $15 \%$ to $50 \%,{ }^{8-17}$ mainly depending on whether so-called minor leaks, defined as a small rupture simply controlled by suction, are excluded or if patients with unruptured aneurysms are included. ${ }^{18}$ IoAR may occur at different stages during surgery including the initial exposure, the dissection of the aneurysm, and the application of the clip (- Fig. 1).$^{19}$ There is considerable controversy over the question of whether ioAR affects clinical outcome ${ }^{17,20}$ and whether it is related to the timing of surgery. ${ }^{12,17}$ This study correlates preoperative clinical and radiographic factors with the occurrence of ioAR, as well as with clinical outcome.

\section{Material and Methods}

\section{Patient Data and Inclusion}

Patient selection was based on a retrospective analysis of our prospective subarachnoid hemorrhage (SAH) patient database collected between January 2008 and August 2012 to analyze ioAR as a primary end point. We included consecutive patients with ruptured saccular aneurysms who underwent microsurgical clipping to occlude the ruptured aneurysms. Patients with no saccular aneurysms, no ruptured aneurysms, aneurysms treated with interventional coiling, or alternative interventional/surgical treatment except surgical clipping were excluded. Our hospital represents a cerebrovascular center with a clipping-to-coiling ratio of $\sim 40$ to $60 \%$ for ruptured aneurysms. Interdisciplinary consent between neuroradiologists and neurosurgeons is obtained in all cases before treatment. Within this time period we treated 282 aSAH patients including 182 coiled and 100 clipped patients.

\section{Chart Data and Radiologic Characteristics}

For all included patients we collected and analyzed the demographic data, cardiovascular risk factors, preoperative radiologic aneurysm characteristics, as well as timing of surgery (time interval between initial bleeding and surgery), and preoperative severity grades (Hunt and Hess [HH], Fisher, and World Federation of Neurological Societies [WFNS]). Cardiovascular risk factors included smoking status, body mass index (BMI), diabetes type 2 (DM), arterial hypertension (aHT), and medication for aHT or DM used before aSAH. Grouping of ioAR or non-ioAR patients was based on information from the surgical report and confirmed by videotapes if available. IoAR was defined as any active arterial bleeding from the aneurysm intended to treat during surgery. Videotapes from patients with ioAR $(n=24)$ were further analyzed to assess surgical technique. For the missing 10 patients, information was obtained from the surgical report only.

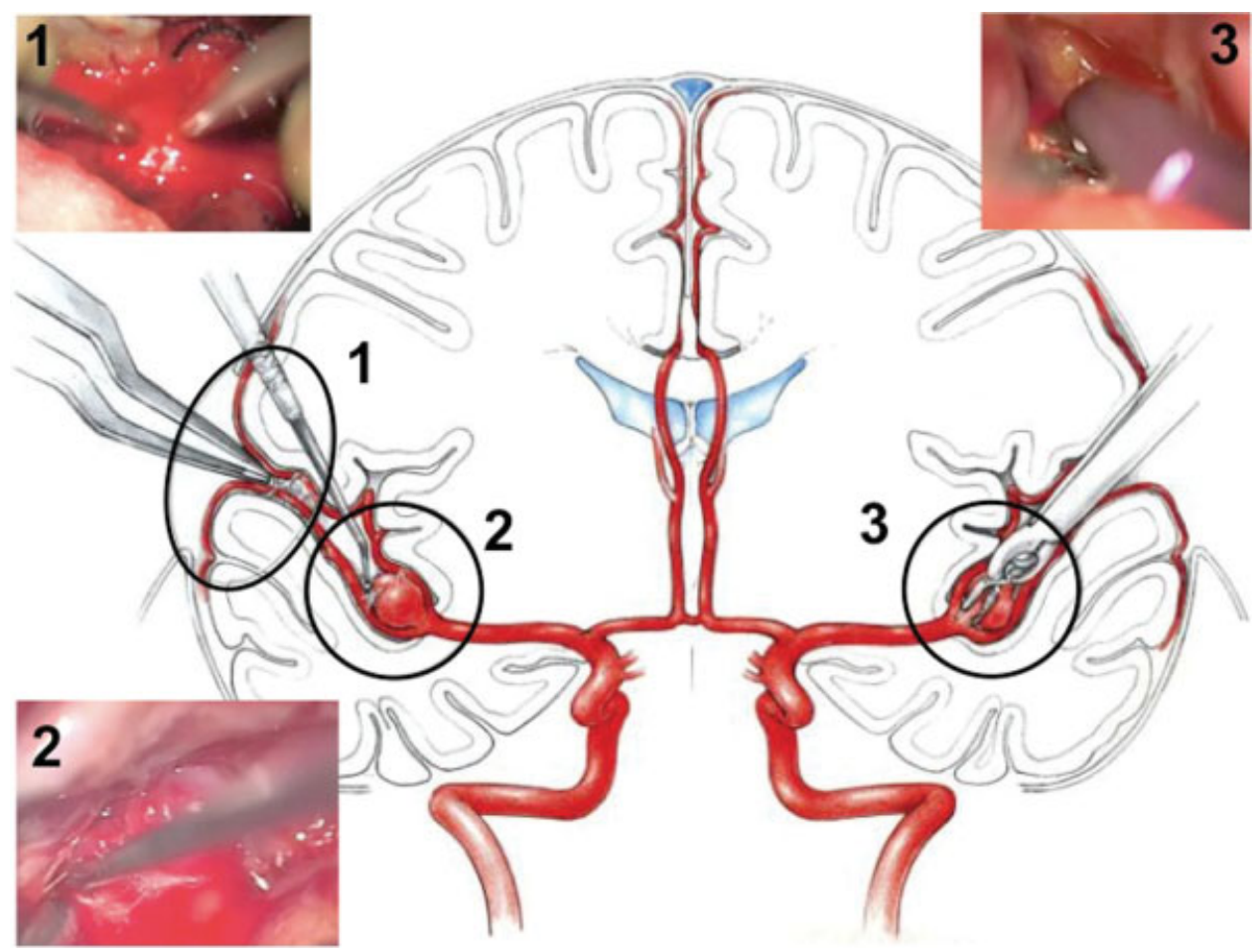

Fig. 1 Illustration and intraoperative microscopic images of the three different stages of intraoperative aneurysm rupture that may occur using a media bifurcation aneurysm as an example: (1) during initial exposure (group A); (2) during the dissection of the aneurysm (group B); and (3) during the application of the clip (group C). 
Radiologic characteristics were based on initial computed tomography (CT) including CT angiography (CTA) or conventional angiography. Aneurysm localization, size, neck diameter, dome-to-neck ratio, maximal cisternal SAH thickness, intraventricular hemorrhage (IVH), and ICH and acute bleeding from the aneurysm, known as the spot sign on CTA, were assessed for all patients. Maximal cisternal SAH thickness was also summarized based on the Barrow Neurological Institute (BNI) score. ${ }^{21}$

The spot sign was defined as an active contrast extravasation on multidetector CTA if the conditions described by Delgado Almandoz et al were fulfilled (one or more spots within the ICH per patient, $\geq 120 \mathrm{HU}$, discontinuous from vasculature, of any size and morphology). ${ }^{22,23}$ An example of a spot sign is depicted in -Supplementary Fig. 1 (online-only). The presence of calcifications was evaluated on native preoperative CT bone windows, and any calcification within the vessel wall was counted as the presence of calcification. Data of all SAH patients treated at our center are prospectively collected including demographics and SAH scores. However, in this study additional parameters such as surgical details by analyzing videos, rated ioARs, and radiographic data, as well as clinical outcomes, were collected retrospectively. Acquisition of patient data was performed in accordance with institutional guidelines and federal law (Ethics Committee no. KEK-StV-Nr. 12/14).

\section{Statistical Analyses}

Scores and continuous variables were expressed as means plus or minus standard deviations and compared between groups using the Mann-Whitney test. Categorical variables were expressed as frequencies with percentages and compared using the chi-square test or Fisher exact test where appropriate. No correction for multiple testing was performed.

Multiple logistic regression was used to analyze predictors of ioAR. Model calibration was assessed using the HosmerLemeshow test. All analyses were performed using IBM SPSS v.20.0 (SPSS Inc., Chicago, Illinois, United States). Two-sided $p$ values $<0.05$ were considered statistically significant.

\section{Results}

\section{Patient Characteristics}

Of 100 patients ( 38 men and 62 women) with a median age of 57.4 years (range: $23-85$ years), ioAR was observed in 34 cases (34\%), and 16 (16 of 34 [47\%]) of these patients presented with an ICH. IoAR occurred during initial exposure $(n=5)$, dissection of the aneurysm $(n=24)$, and during or after clipping ( $n=5$; - Fig. $\mathbf{1})$. In the initial exposure ioAR group, in four of five cases the aneurysm ruptured during ICH evacuation. The other 12 patients with ioAR and ICH did not show an aneurysm rupture during ICH evacuation. None of the patients showed clinical or radiologic signs of rebleeding between hospital admission and the start of the surgical intervention. Demographic data and cardiovascular patient risk factors including smoking, BMI, DM, and aHT were not significantly different between the two groups ( - Table $\mathbf{1}$ ).

\section{Aneurysm Characteristics and Radiologic Assessment}

In our patient population, most aneurysms were located in the middle cerebral artery (MCA) $(n=38)$, anterior communicating artery $(\mathrm{AComA})(n=29)$, or internal carotid artery (ICA) $(n=12)$ with the highest ioAR rate present in the ICA (42\%) and AComA (41\%) (-Table 2). Size, neck diameter, dome-to-neck ratio, size of SAH, presence of calcifications, and IVH were not significantly different between the ioAR and non-ioAR group. ICH occurred in a total of 31 patients (31\%) and was significantly more frequent in the group of patients subsequently developing ioAR (16 of 34 [47\%]) compared with the non-ioAR group (15 of 66 [23\%]; $p=0.013$ ); a high volume of ICH was associated with an intraoperative rupture $(p=0.017$; - Table 3$)$. Of the 31 patients with ICH, 7 patients (23\%) displayed a spot sign on preoperative CTA, and all patients with a preoperative spot sign had an ioAR. The spot sign represented a significant risk factor for ioAR $(p=0.007)$.

\section{SAH Scores}

Severe SAH cases were overrepresented in our series: 27 patients had a WFNS score of 5 , and 14 patients had a WFNS score of 4 (severe SAH in $41 \%$ of the cases). The initial clinical status was significantly worse in patients with ioAR (Glasgow Coma Scale score $p=0.022$; WFNS $p=0.023$; and $\mathrm{HH}$ $p=0.002)$, which was also confirmed by a high Fisher grade in these patients $(p=0.012)$ (-Figs. 2 and 3). However, maximal cisternal SAH thickness, summarized in the BNI score, was not expedient as a predictive score for ioAR $(p=0.97)$ (-Fig. 3). The maximal cisternal SAH thickness was also not associated with a significant risk for ioAR.

Table 1 Baseline characteristics and cardiovascular risk factors

\begin{tabular}{|l|l|l|l|l|}
\hline Variable & $\begin{array}{l}\text { All } \\
(\boldsymbol{n}=\mathbf{1 0 0})\end{array}$ & $\begin{array}{l}\text { Non-ioAR } \\
(\boldsymbol{n}=\mathbf{6 6})\end{array}$ & IoAR $(\boldsymbol{n}=\mathbf{3 4 )}$ & $\boldsymbol{p}$ value \\
\hline Age, mean \pm SD, y & $57.4 \pm 13.9$ & $58.2 \pm 14.3$ & $55.9 \pm 13.1$ & 0.46 \\
\hline Gender, number (\% male) & $38(38)$ & $27(41)$ & $11(32)$ & 0.51 \\
\hline BMl, mean \pm SD, kg/m ${ }^{2}$ & $24.3 \pm 3.8$ & $24.4 \pm 4.3$ & $24.1 \pm 3.0$ & 0.86 \\
\hline Diabetes mellitus type 2, $n$ (\%) & $3(3)$ & $1(2)$ & $2(6)$ & 0.11 \\
\hline Arterial hypertension, $n(\%)$ & $36(36)$ & $21(32)$ & $15(44)$ & 0.27 \\
\hline Smoking, $n(\%)$ & $37(37)$ & $26(40)$ & $11(32)$ & 0.35 \\
\hline
\end{tabular}

Abbreviations: BMI, body mass index; IoAR, intraoperative aneurysm rupture; SD, standard deviation. 
Table 2 Aneurysm localization and intraoperative aneurysm rupture rate

\begin{tabular}{|l|l|l|l|}
\hline Aneurysm location & No. of aneurysms, $\boldsymbol{n}=\mathbf{1 0 0}$ & No. of ioARs, $\boldsymbol{n}=\mathbf{3 4}$ (\% of total number) & loAR rate (\%) \\
\hline MCA & 38 & $15(44)$ & 40 \\
\hline AComA & 29 & $12(35)$ & 41 \\
\hline ICA & 12 & $5(15)$ & 42 \\
\hline PComA & 7 & $2(6)$ & 29 \\
\hline PICA & 5 & - & - \\
\hline Pericallosal & 3 & - & - \\
\hline Anterior choroidal & 3 & - & - \\
\hline PCA & 3 & - & - \\
\hline
\end{tabular}

Abbreviations: AComA, anterior communicating artery; ICA, internal carotid artery; IoAR, intraoperative aneurysm rupture; MCA, middle cerebral artery; PCA, posterior cerebral artery; PComA, posterior communicating artery; PICA, posterior inferior cerebellar artery.

\section{Surgical Characteristics}

Day of surgery ( $p=0.76)$ and preoperative placement of an external ventricular drain $(p=0.35)$ or lumbar drain $(p=0.37)$ were not significantly associated with ioAR (-Table 4). Patients with ioAR were divided into three subgroups based on the time point of the aneurysm rupture during surgery (group A, rupture at initial exposure; group B, rupture during dissection of the aneurysm; group $C$, rupture during or after clip application). - Table 5 summarizes the surgical details in the ioAR group among the three subgroups. Interestingly, all patients with an ioAR from group A had MCA aneurysms and a high rate of ICH (four of five [80\%]). Regarding all 100 cases, temporary clipping before definite clipping was rarely performed in nine cases in the ioAR group (-Table 5). However, it was performed in 4 patients (4 of 5) after ioAR in group A, in 17 patients (17 of 24 [71\%]) in group $B$, and 3 patients ( 3 of 5 ) in group C. ICH and the spot sign were more likely present in groups A and B (-Table 5 ).

\section{Clinical Outcome}

With a mean follow-up of 17.6 ( \pm 16.6$)$ months, there was a comparable clinical outcome in all patients, and ioAR did not have a significant impact on clinical outcome (-Fig. 4). Overall mortality was $13 \%$ (12\% in the non-ioAR versus $15 \%$ in the ioAR group; $p=0.82$ ), and patients with a Glasgow Outcome Scale (GOS) score $\leq 3$ were present in $41 \%$ (39\% in the non-ioAR versus $44.1 \%$ in the ioAR group). Patients with ICH and the spot sign had a GOS $\leq 3$ in $71 \%$ (five of seven). Delayed cerebral ischemia (36\% versus 38\%; $p=0.51$ ) and shunt dependency ( $32 \%$ versus $44 \% ; p=0.23$ ) were comparable between the non-ioAR and ioAR group.

\section{Multivariate Analysis}

Variables significant in univariate analyses were included in a multiple logistic regression. The spot sign was not included because it was only available for patients with $\mathrm{ICH}$. We included $\mathrm{ICH}$, WFNS, $\mathrm{HH}$, and Fisher grades. $\mathrm{HH}$ was the only significant predictor of ioAR ( $p=0.03$; odds ratio: 2.3 ; $95 \%$ confidence interval, 1.1-5.0). The results of this analysis are shown in - Table 6 .

\section{Discussion}

The International Subarachnoid Aneurysm Trial (ISAT) led to a shift in treatment recommendations for ruptured intracerebral aneurysms toward endovascular coiling. ${ }^{24}$ All published studies on ioAR analyzed data from before the ISAT results ${ }^{17,18,20}$ Our study included only patients operated in

Table 3 Radiologic aneurysm and hemorrhage characteristics

\begin{tabular}{|c|c|c|c|c|}
\hline Variable & $\begin{array}{l}\text { All } \\
(n=100)\end{array}$ & $\begin{array}{l}\text { Non-ioAR } \\
(n=66)\end{array}$ & IoAR $(n=34)$ & $p$ value \\
\hline Aneurysm size, mean $\pm \mathrm{SD}$, mm & $7.5 \pm 5.9$ & $6.7 \pm 5$ & $9.2 \pm 7.2$ & 0.07 \\
\hline Neck diameter, mean \pm SD, mm & $3.1 \pm 1.6$ & $2.9 \pm 1.2$ & 3.5 & 0.11 \\
\hline Dome-to-neck ratio, mean \pm SD & $2.5 \pm 1.9$ & $2.4 \pm 1.8$ & $2.8 \pm 2.1$ & 0.42 \\
\hline Size $S A H$, mean $\pm S D$, mm & $8.8 \pm 4.9$ & $8.9 \pm 5.2$ & $8.5 \pm 4.1$ & 0.79 \\
\hline $\mathrm{IVH}, n(\%)$ & $38(38)$ & $25(38)$ & $13(38)$ & 0.57 \\
\hline $\mathrm{ICH}, n(\%)$ & $31(31)$ & $15(23)$ & $16(47)$ & 0.013 \\
\hline Volume $\mathrm{ICH}$, mean $\pm \mathrm{SD}, \mathrm{mm}^{3}$ & $4,882 \pm 11,967$ & $3,306 \pm 8,689$ & $7,940 \pm 16,319$ & 0.017 \\
\hline Spot sign, no. of patients (\%) & $7(7)$ & 0 & $7(21)$ & 0.007 \\
\hline
\end{tabular}

Abbreviations: ICH, intracerebral hemorrhage; IoAR, intraoperative aneurysm rupture; IVH, intraventricular hemorrhage; SAH, subarachnoid hemorrhage; SD, standard deviation. 

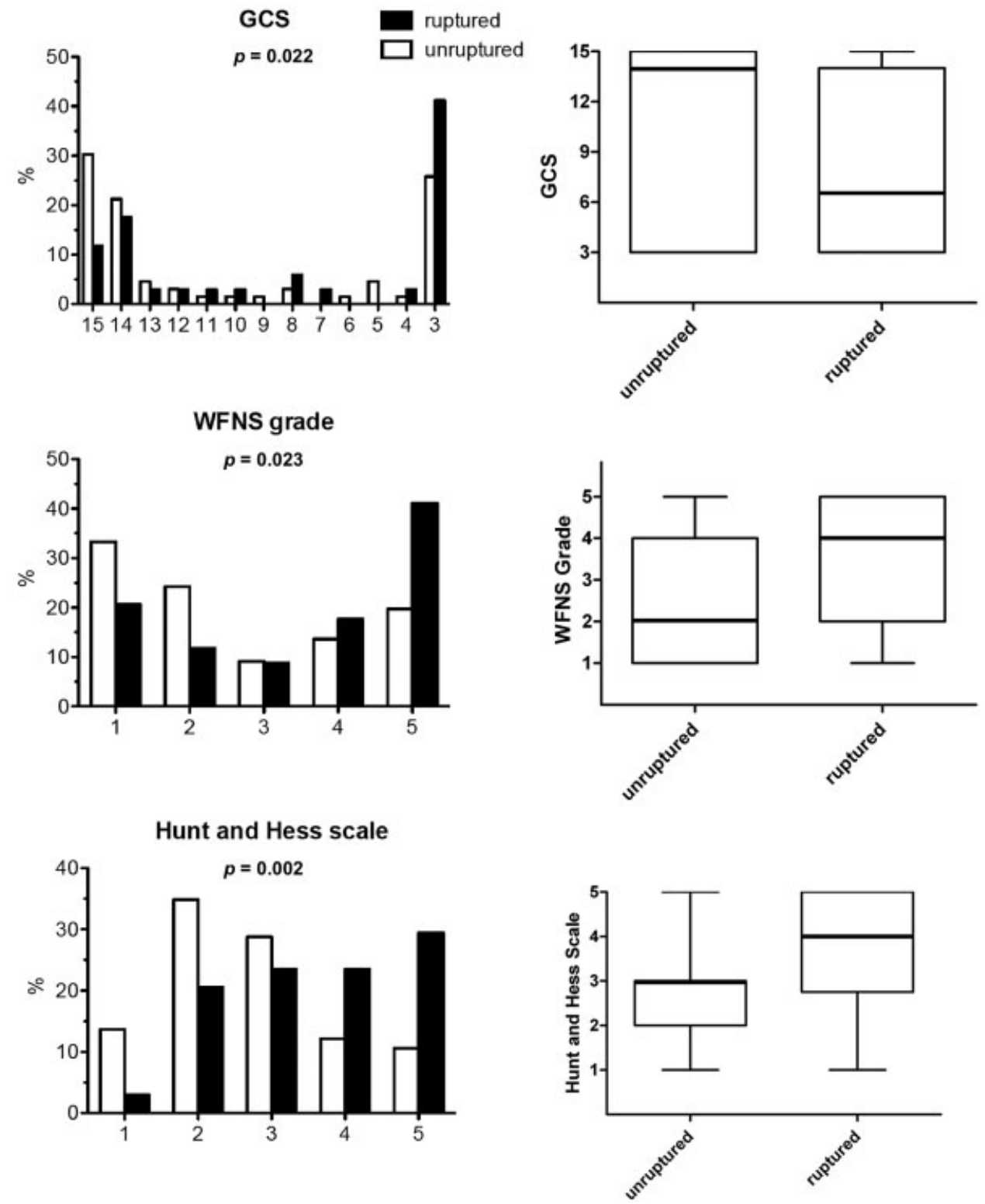

Fig. 2 Distribution (left) and box plot (right) with medians (thick black line) of clinical scores including Glasgow Coma Scale (GCS) score, World Federation of Neurological Societies [WFNS], and Hunt and Hess $(\mathrm{HH})$ for the patient group with and without intraoperative aneurysm rupture. The $p$ value is based on the Mann-Whitney test.

the post-ISAT era and therefore more reliably reflects the currently treated SAH patient population.

The aim of the study was to evaluate preoperative clinical and radiographic risk factors as well as the timing of surgery to assess the incidence of ioAR rupture and its influence on clinical outcome. Our ioAR rate of $34 \%$ was in the higher range compared with previously published studies, which is most likely due to the inclusion of any intraoperative aneurysm leakage/rupture event (in contrast to previous studies, we also included small leaks that were easily controlled surgically). We do not believe the rupture rate is biased due to the retrospective design. With a coiling rate of $\sim 60 \%$ at our center, another reason for the high ioAR rate in our series might be the overrepresentation of complex cases in our surgical cohort (high severity grades and high rate of ICH). However, this might be an adequate representation of a surgical cohort in today's post-ISAT era.

Preoperative clinical status, as summarized by higher $\mathrm{HH}$ and WFNS grading scores, as well as the presence of ICH and the spot sign, were the only statistically significant predictors for ioAR in univariate analyses. In a multivariate model, $\mathrm{HH}$ was the only significant independent factor. This is most likely explained by the interdependence of factors that reflect initial clinical status (i.e., WFNS and $\mathrm{HH}$ show a strong positive correlation, and ICH influences both $\mathrm{HH}$ and WFNS). Any other radiographic aneurysmal characteristics, timing of surgery, or any kind of cerebrospinal fluid draining before treatment were not associated 


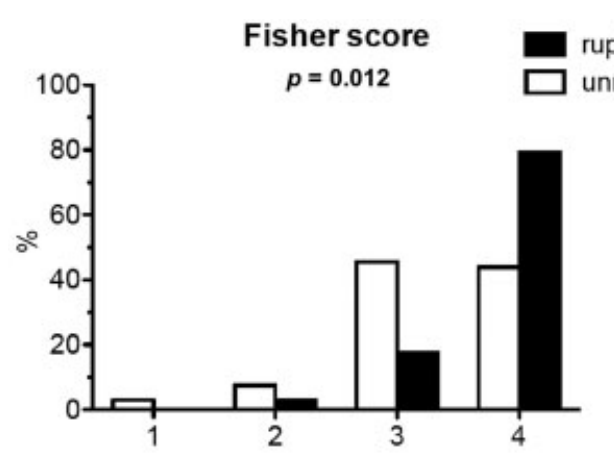

ruptured

unruptured
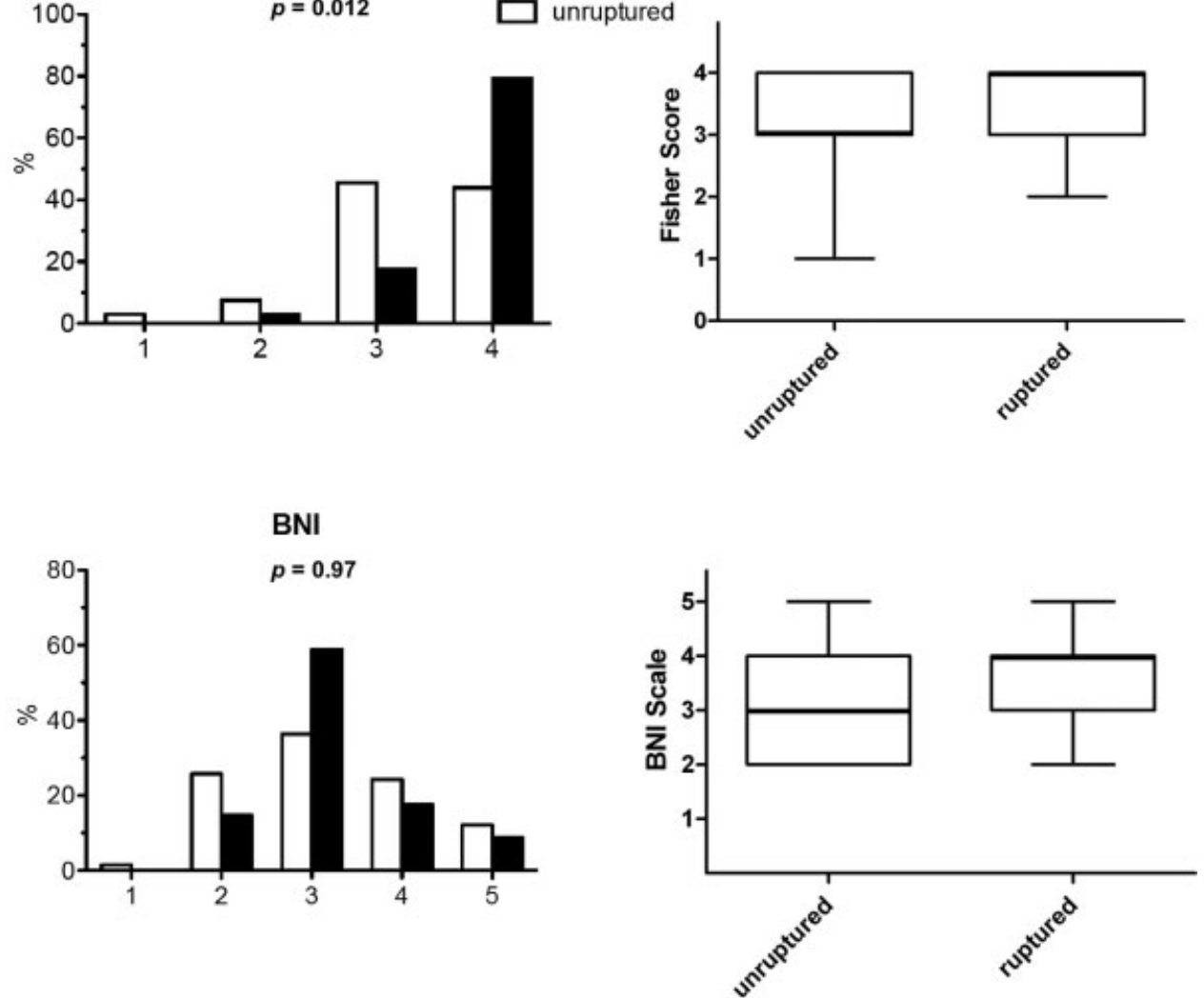

Fig. 3 Distribution (left) and box plot (right) with medians (thick black line) of the Fisher score and Barrow Neurological Institute (BNI) score for the patient group without and with intraoperative aneurysm rupture. The $p$ value is based on the Mann-Whitney test.

with ioAR. Clinical outcome, delayed cerebral ischemia, as well as shunt dependency, were comparable in all patient groups.

The most striking and novel result of our study pertains to radiologic predictors of ioAR. Patients presenting with ICH on the initial CT scan had a significantly higher risk of ioAR. We also found within the ICH group that all patients with a spot sign $(n=7)$ had an ioAR. Interestingly, there was no difference in outcome between patients with or without ioAR and between patients with or without the spot sign. In a 2013 publication, Brouwers et al showed that in patients with aSAH, the rate of spot signs in ICH is lower than in primary $\mathrm{ICH}^{23}$ They also showed the lack of association between the spot sign and clinical outcome. ${ }^{23}$ These results accord well with our findings.

Finally, ioAR patients showed a statistically significant worse initial clinical status compared with non-ioAR patients. No other factors such as cardiovascular risk factors or other

Table 4 Surgical details of the ioAR and non-ioAR groups

\begin{tabular}{|c|c|c|c|}
\hline & IoAR, $n=34(\%)^{a}$ & Non-ioAR, $n=66(\%)^{b}$ & $p$ value \\
\hline \multicolumn{4}{|l|}{ Day of surgery } \\
\hline 0 & $10(29)$ & $17(26)$ & \multirow[t]{5}{*}{0.76} \\
\hline 1 & $13(38)$ & $29(44)$ & \\
\hline 2 & $4(12)$ & $7(11)$ & \\
\hline 3 & $4(12)$ & $2(3)$ & \\
\hline$>3$ & $3(9)$ & $11(17)$ & \\
\hline EVD before clipping & $5(15)$ & $12(18)$ & 0.35 \\
\hline LP/LD before clipping & $3(9)$ & $10(15)$ & 0.37 \\
\hline
\end{tabular}

Abbreviations: EVD, external ventricular drain; IoAR, intraoperative aneurysm rupture; LD, lumbar drain; LP, lumbar puncture.

a Percentages in this row refer to $n=34$.

${ }^{\mathrm{b}}$ Percentages in this row refer to $n=66$. 
Table 5 Surgical characteristics of the three intraoperative aneurysm rupture groups

\begin{tabular}{|c|c|c|c|}
\hline Type of ioAR & $\begin{array}{l}\text { Initial exposure } \\
n=5\end{array}$ & $\begin{array}{l}\text { Dissection of aneurysm } \\
n=24(\%)^{\mathrm{a}}\end{array}$ & $\begin{array}{l}\text { Clip application } \\
n=5\end{array}$ \\
\hline MCA $(n=15)$ & 5 & $9(37)$ & 1 \\
\hline AcomA $(n=12)$ & - & $10(42)$ & 2 \\
\hline ICA $(n=5)$ & - & $4(17)$ & 1 \\
\hline PComA $(n=2)$ & - & $1(4)$ & 1 \\
\hline Temporary occlusion before ioAR & 0 & $8(33)$ & 1 \\
\hline Temporary occlusion after ioAR & 4 & $17(71)$ & 3 \\
\hline Preparation of aneurysm, blunt & - & $13(54)$ & 4 \\
\hline Location of rupture, neck & 1 & $13(54)$ & 3 \\
\hline \multicolumn{4}{|l|}{ No. of definite clips } \\
\hline 1 & 5 & $20(83)$ & 0 \\
\hline 2 & 0 & $4(7)$ & 4 \\
\hline$>2$ & 0 & 0 & 1 \\
\hline $\mathrm{ICH}(n=16)$ & 4 & $10(42)$ & 2 \\
\hline Spot sign $(n=7)$ & 2 & $5(21)$ & 0 \\
\hline
\end{tabular}

Abbreviations: AComA, anterior communicating artery; ICA, internal carotid artery; ICH, intracerebral hemorrhage; loAR, intraoperative aneurysm rupture; MCA, middle cerebral artery; PComA, posterior communicating artery.

apercentages in this row refer to $n=24$.

radiographic aneurysm characteristics (such as aneurysm size or shape of aneurysm) were associated with ioAR. These factors need to be further analyzed in a prospective trial. Our findings are in line with previous observations and follow a logical pattern because patients with an ICH and active bleeding most likely present with a reduced initial clinical status due to the hemorrhagic mass lesion. It has to be considered that the initial clinical status in the ioAR group was significantly worse. Taking into consideration that final clinical outcome was not significantly different between both groups, one might come to the somewhat paradoxical conclusion that ioAR had a protective effect. However, the mass effect in patients with ICH and the active bleeding in subjects with a positive spot sign in the ioAR group could be one relevant factor contributing to poor initial clinical status as mentioned earlier, which then again could be partially reversed by surgical intervention.

It is well established that the spot sign indicates active bleeding and predicts hematoma extension as well as a poor outcome in patients with spontaneous ICH. $22,23,25,26$ Our study shows by analogy that the spot sign is associated with the risk of aneurysm re-rupture and active bleeding intraoperatively.

ICH and spot signs were factors associated with surgical technique because most intraoperative ruptures in our study occurred during initial exposure (group A) and therefore

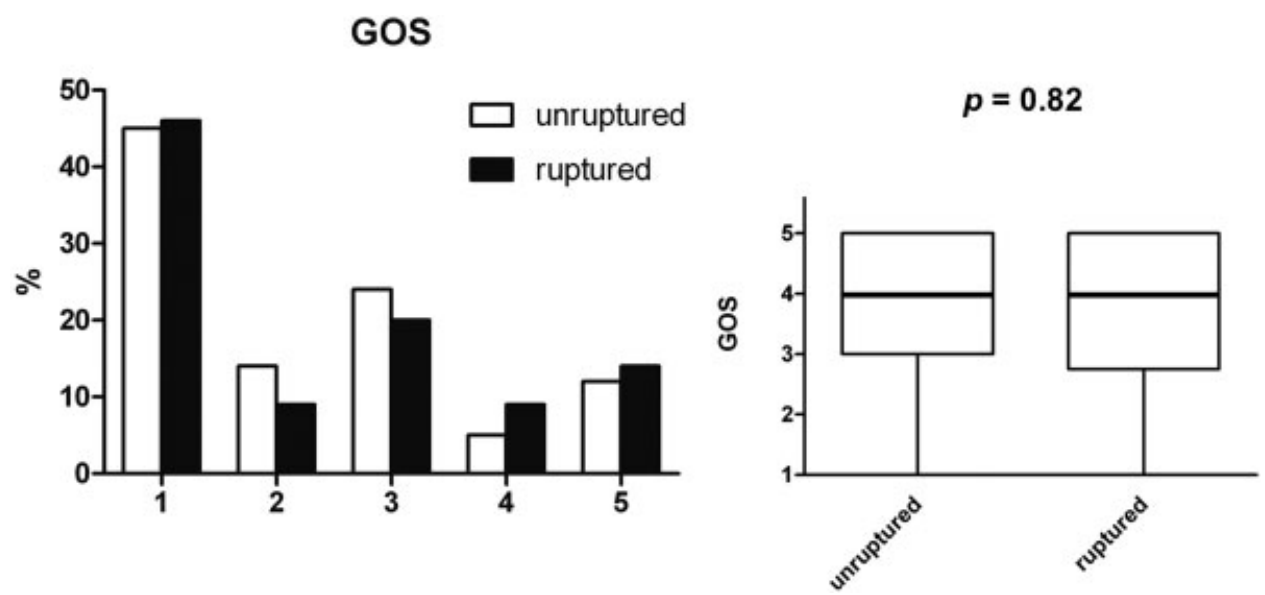

Fig. 4 Distribution (left) and box plot (right) with medians (thick black line) of Glasgow Outcome Scale (GOS) score for the patient group without and with intraoperative aneurysm rupture. The $p$ value is based on the Mann-Whitney test. 
Table 6 Multivariate analysis to analyze predictors of intraoperative aneurysm rupture

\begin{tabular}{|l|l|l|l|l|}
\hline Variable & OR & Lower 95\% Cl & Upper 95\% Cl & $p$ \\
\hline ICH & 1.3 & 0.4 & 4.3 & 0.65 \\
\hline Hunt and Hess & 2.2 & 1.1 & 5.0 & 0.03 \\
\hline WFNS & 0.7 & 0.4 & 1.2 & 0.22 \\
\hline Fisher & 1.8 & 0.7 & 4.3 & 0.21 \\
\hline
\end{tabular}

Abbreviations: ICH, intracranial hemorrhage; $\mathrm{Cl}$, confidence interval; OR, odds ratio; WFNS, World Federation of Neurological Societies (grade).

before dissection of the aneurysm. ICH was a significant factor in the univariate analysis but not in the multivariate model. However, none of the patients with a spot sign and only two patients with ICH presented ioAR during or after clip application (group C). Aneurysm rupture in group $C$ seems more likely related to the surgical technique than the other two groups.

Temporary clipping is another important factor discussed in the literature. ${ }^{27-30}$ A recent prospective study showed that the use of elective temporary clipping decreased the rate of ioAR leading to improved outcome, whereas repeated rescue clipping in case of ioAR and total clipping time of at least 20 minutes had a significant impact on outcome. ${ }^{28}$ In our study, patients with ioAR were rarely temporarily clipped before rupture. However, due to the retrospective nature of this study, only limited information about temporary occlusion was available for all patients (length and type), and we could not fully determine the degree to which the surgeons' judgment affected the use of temporary clipping. This could be further analyzed in a prospective study.

With regard to clinical outcome, we found similar results for both groups without and with ioAR. In accordance with this finding, Sandalcioglu et al reported comparable outcomes for both groups with a trend to increased morbidity and mortality when ioAR occurred in patients with a poor initial condition. ${ }^{20}$ All these results show that ioAR does not significantly impact clinical outcome, if handled in experienced hands, ${ }^{20,31}$ because advanced microsurgical techniques allow early control of an intraoperative rupture event. Complications of SAH such as delayed cerebral ischemia or shunt dependency did not depend on the occurrence of ioAR. Even though clinical outcome was not affected by ioAR in our series ( $12 \%$ in the non-ioAR versus $15 \%$ in the ioAR group) and in the microsurgical clipping literature, there is good evidence that ioAR during endovascular interventional procedures has a deleterious impact on outcome. In a series by Guglielmi et al, most of the patients died following ioAR during coiling of aneurysms of the posterior circulation. ${ }^{32}$ However, this study showed data in the beginning of coiling without modern microcatheters and without balloon-assisted coiling methods.

The more recent published Cerebral Aneurysm Rerupture After Treatment (CARAT) study analyzed 1,010 patients (299 coiled and 711 clipped) and found in case of aneurysm rupture a periprocedural mortality rate of $31 \%$ among those clipped compared with $63 \%$ within the coiling group. ${ }^{33}$ In our opinion, this difference is best explained by superior measures of bleeding control in open surgery compared with the limited access during interventional procedures. Modern balloon-assisted coiling methods might have an impact, but no data are available yet. Interestingly, the rate of intraprocedural re-rupture during coiling in the CARAT series was lower (5\%) compared with the clipping group (19\%). Based on the CARAT data, one may speculate that the lower intraprocedural rupture rate may also reflect a selection bias: unstable aneurysms with ICH are more likely to undergo surgical clipping because of the mass effect. The results of our study may suggest that unstable aneurysms (defined by ICH and a positive spot sign in our study) might profit from surgical clipping because of the increased risk of intraprocedural rupture. However this needs to be further analyzed in a prospective study including both surgical as well as endovascular treated aSAH patients.

\section{Limitations}

Our study is mainly limited by its retrospective design and by analyzing a pure surgical cohort. The pathophysiologic mechanism underlying the association of a positive spot sign and increased risk for ioAR in our study population remains unclear. One might hypothesize that in ICH with a spot sign, active bleeding prevents clot stabilization around the aneurysm neck. And vice versa, in patients without active bleeding the clot around the aneurysm neck has more time to stabilize and mature without being disrupted by active (re) bleeding. In addition, the local consumption of clotting factors during active bleeding right before surgery might contribute to a local hypocoagulative state. However, these are only hypotheses and not backed up by our study data. Larger prospective studies involving both surgical and endovascular patients are needed.

\section{Summary}

$\mathrm{HH}$ grade and the spot sign were associated with ioAR during microsurgical clipping of a ruptured aneurysm. However, there was no difference regarding clinical outcome and other complications between the two groups. ICH and the spot sign seem to predict an unstable aneurysm, and ioAR should be anticipated and reflected in surgical strategy such as surgical approach, temporary clipping, and anesthesiological measures. 


\section{References}

1 Lloyd-Jones D, Adams R, Carnethon M, et al; American Heart Association Statistics Committee and Stroke Statistics Subcommittee. Heart disease and stroke statistics-2009 update: a report from the American Heart Association Statistics Committee and Stroke Statistics Subcommittee. Circulation 2009;119(3):480-486

2 Feigin VL, Lawes CM, Bennett DA, Anderson CS. Stroke epidemiology: a review of population-based studies of incidence, prevalence, and case-fatality in the late 20th century. Lancet Neurol 2003;2(1):43-53

3 Suarez JI, Tarr RW, Selman WR. Aneurysmal subarachnoid hemorrhage. N Engl J Med 2006;354(4):387-396

4 van Gijn J, Rinkel GJ. Subarachnoid haemorrhage: diagnosis, causes and management. Brain 2001;124(Pt 2):249-278

5 Johnston SC, Selvin S, Gress DR. The burden, trends, and demographics of mortality from subarachnoid hemorrhage. Neurology 1998;50(5):1413-1418

6 Nieuwkamp DJ, Setz LE, Algra A, Linn FH, de Rooij NK, Rinkel GJ. Changes in case fatality of aneurysmal subarachnoid haemorrhage over time, according to age, sex, and region: a meta-analysis. Lancet Neurol 2009;8(7):635-642

7 Larsen CC, Astrup J. Rebleeding after aneurysmal subarachnoid hemorrhage: a literature review. World Neurosurg 2013;79(2): 307-312

8 Batjer H, Samson D. Intraoperative aneurysmal rupture: incidence, outcome, and suggestions for surgical management. Neurosurgery 1986;18(6):701-707

9 Giannotta SL, Oppenheimer JH, Levy ML, Zelman V. Management of intraoperative rupture of aneurysm without hypotension. Neurosurgery 1991;28(4):531-535; discussion 535-536

10 Hillman J, Säveland H, Jakobsson KE, et al. Overall management outcome of ruptured posterior fossa aneurysms. J Neurosurg 1996;85(1):33-38

11 Jomin M, Lesoin F, Lozes G. Prognosis with 500 ruptured and operated intracranial arterial aneurysms. Surg Neurol 1984;21(1): 13-18

12 Kassell NF, Boarini DJ, Adams HP Jr, et al. Overall management of ruptured aneurysm: comparison of early and late operation. Neurosurgery 1981;9(2):120-128

13 Le Roux PD, Elliot JP, Newell DW, Grady MS, Winn HR. The incidence of surgical complications is similar in good and poor grade patients undergoing repair of ruptured anterior circulation aneurysms: a retrospective review of 355 patients. Neurosurgery 1996;38(5):887-893; discussion 893-895

14 Le Roux PD, Elliott JP, Downey L, et al. Improved outcome after rupture of anterior circulation aneurysms: a retrospective 10-year review of 224 good-grade patients. J Neurosurg 1995;83(3): 394-402

15 Rinne J, Hernesniemi J, Niskanen M, Vapalahti M. Management outcome for multiple intracranial aneurysms. Neurosurgery 1995; 36(1):31-37; discussion 37-38

16 Samson DS, Hodosh RM, Reid WR, Beyer CW, Clark WK. Risk of intracranial aneurysm surgery in the good grade patient: early versus late operation. Neurosurgery 1979;5(4):422-426

17 Schramm J, Cedzich C. Outcome and management of intraoperative aneurysm rupture. Surg Neurol 1993;40(1):26-30

18 Leipzig TJ, Morgan J, Horner TG, Payner T, Redelman K, Johnson CS. Analysis of intraoperative rupture in the surgical treatment of 1694 saccular aneurysms. Neurosurgery 2005;56(3):455-468; discussion $455-468$
19 Batjer H, Samson DS. Management of intraoperative aneurysm rupture. Clin Neurosurg 1990;36:275-288

20 Sandalcioglu IE, Schoch B, Regel JP, et al. Does intraoperative aneurysm rupture influence outcome? Analysis of 169 patients. Clin Neurol Neurosurg 2004;106(2):88-92

21 Wilson DA, Nakaji P, Abla AA, et al. A simple and quantitative method to predict symptomatic vasospasm after subarachnoid hemorrhage based on computed tomography: beyond the Fisher scale. Neurosurgery 2012;71(4):869-875

22 Delgado Almandoz JE, Yoo AJ, Stone MJ, et al. Systematic characterization of the computed tomography angiography spot sign in primary intracerebral hemorrhage identifies patients at highest risk for hematoma expansion: the spot sign score. Stroke 2009; 40(9):2994-3000

23 Brouwers HB, Backes D, Kimberly WT, et al. Computed tomography angiography spot sign does not predict case fatality in aneurysmal subarachnoid hemorrhage with intraparenchymal extension. Stroke 2013;44(6):1590-1594

24 Molyneux AJ, Kerr RS, Yu LM, et al; International Subarachnoid Aneurysm Trial (ISAT) Collaborative Group. International subarachnoid aneurysm trial (ISAT) of neurosurgical clipping versus endovascular coiling in 2143 patients with ruptured intracranial aneurysms: a randomised comparison of effects on survival, dependency, seizures, rebleeding, subgroups, and aneurysm occlusion. Lancet 2005;366(9488):809-817

25 Delgado Almandoz JE, Kelly HR, Schaefer PW, et al. CT angiography spot sign predicts in-hospital mortality in patients with secondary intracerebral hemorrhage. J Neurointerv Surg 2012;4(6):442-447

26 Havsteen I, Ovesen C, Christensen AF, Hansen CK, Nielsen JK, Christensen $\mathrm{H}$. Showing no spot sign is a strong predictor of independent living after intracerebral haemorrhage. Cerebrovasc Dis 2014;37(3):164-170

27 Charbel FT, Ausman JI, Diaz FG, Malik GM, Dujovny M, Sanders J. Temporary clipping in aneurysm surgery: technique and results. Surg Neurol 1991;36(2):83-90

28 Dhandapani S, Pal SS, Gupta SK, Mohindra S, Chhabra R, Malhotra SK. Does the impact of elective temporary clipping on intraoperative rupture really influence neurological outcome after surgery for ruptured anterior circulation aneurysms?-a prospective multivariate study Acta Neurochir (Wien) 2013;155(2): 237-246

29 Griessenauer CJ, Poston TL, Shoja MM, et al. The impact of temporary artery occlusion during intracranial aneurysm surgery on long-term clinical outcome: part I. Patients with subarachnoid hemorrhage. World Neurosurg 2014;82(1-2):140-148

30 Woertgen C, Rothoerl RD, Albert R, Schebesch KM, Ullrich OW. Effects of temporary clipping during aneurysm surgery. Neurol Res 2008;30(5):542-546

31 van Lindert EJ, Böcher-Schwarz HG, Perneczky A. The influence of surgical experience on the rate of intraoperative aneurysm rupture and its impact on aneurysm treatment outcome. Surg Neurol 2001;56(3):151-156; discussion 156-158

32 Guglielmi G, Viñuela F, Duckwiler G, et al. Endovascular treatment of posterior circulation aneurysms by electrothrombosis using electrically detachable coils. J Neurosurg 1992;77(4):515-524

33 Elijovich L, Higashida RT, Lawton MT, Duckwiler G, Giannotta S, Johnston SC; Cerebral Aneurysm Rerupture After Treatment (CARAT) Investigators. Predictors and outcomes of intraprocedural rupture in patients treated for ruptured intracranial aneurysms: the CARAT study. Stroke 2008;39(5):1501-1506 